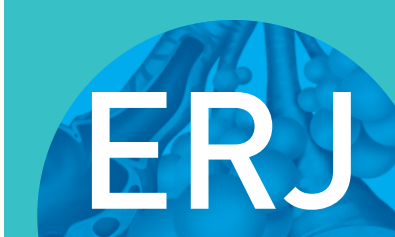

open research
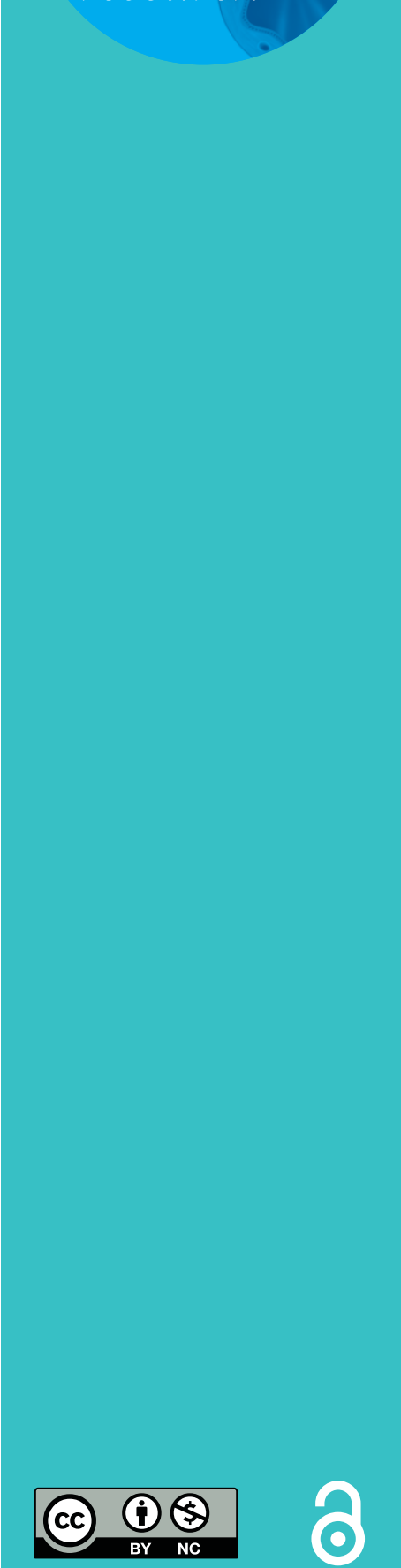

\section{Biomarkers for tuberculosis: the case for lipoarabinomannan}

\author{
Margarida Correia-Neves ${ }^{1,2,3}$, Gabrielle Fröberg (103,4, Liudmyla Korshun ${ }^{5}$, \\ Sofia Viegas ${ }^{6}$, Paula Vaz ${ }^{6,7}$, Nehaben Ramanlal $^{7}$, Judith Bruchfeld ${ }^{3,4}$, \\ Beston Hamasur ${ }^{5,8}$, Patrick Brennan ${ }^{9}$ and Gunilla Källenius (1) ${ }^{3}$
}

Affiliations: 'Life and Health Sciences Research Institute (ICVS), School of Medicine, University of Minho, Braga, Portugal. ${ }^{2}$ ICVS/3B's, PT Government Associate Laboratory, Braga/Guimarães, Portugal. ${ }^{3}$ Division of Infectious Diseases, Dept of Medicine Solna, Karolinska Institutet, Stockholm, Sweden. ${ }^{4}$ Dept of Infectious Diseases, Karolinska University Hospital, Stockholm, Sweden. ${ }^{5}$ Biopromic AB, Solna, Sweden. ${ }^{6}$ Instituto Nacional de Saúde, Ministério da Saúde, Maputo, Mozambique. ${ }^{7}$ Fundação Ariel Glaser Contra o SIDA Pediátrico, Maputo, Mozambique. ${ }^{8}$ Dept of Microbiology, Tumor and Cell Biology, Karolinska Institutet, Stockholm, Sweden. ${ }^{9}$ Dept of Microbiology, Immunology and Pathology, Colorado State University, Fort Collins, CO, USA.

Correspondence: Gunilla Källenius, Division of Infectious Diseases, Dept of Medicine Solna, Karolinska Institutet, Stockholm 17176, Sweden. E-mail: gunilla.kalleniusaki.se

ABSTRACT Tuberculosis (TB) is considered the most onerous of infectious diseases according to recent reports from the World Health Organization. Available tests for TB diagnosis present severe limitations, and a reliable point-of-care (POC) diagnostic test does not exist. Neither is there a test to discern between the different stages of TB, and in particular to predict which patients with Mycobacterium tuberculosis infection and no clinical signs are more at risk of advancing to overt disease. We here review the usefulness of mycobacterial lipoarabinomannan (LAM) as a diagnostic marker for active and latent TB and, also, aspects of the immune response to LAM relevant to such tests. There is a high potential for urinary LAM-based POC tests for the diagnosis of active TB. Some technical challenges to optimised sensitivity of the test will be detailed. A method to quantify LAM in urine or serum should be further explored as a test of treatment effect. Recent data on the immune response to LAM suggest that markers for host response to LAM should be investigated for a prognostic test to recognise individuals at the greatest risk of disease activation.

@ERSpublications

There is a high potential for a urinary LAM-based point-of-care test to diagnose TB. Markers for host response to LAM should be explored to identify those at highest risk of developing active TB. http://ow.ly/FyCs30n4uFE

Cite this article as: Correia-Neves M, Fröberg G, Korshun L, et al. Biomarkers for tuberculosis: the case for lipoarabinomannan. ERJ Open Res 2019; 5: 00115-2018 [https://doi.org/10.1183/ 23120541.00115-2018].

Received: July 202018 | Accepted after revision: Nov 052018

Copyright $\odot$ ERS 2019. This article is open access and distributed under the terms of the Creative Commons Attribution Non-Commercial Licence 4.0. 
Tuberculosis: a global threat to human health

It is estimated that about $23 \%$ of the global population is infected with Mycobacterium tuberculosis [1] and tuberculosis (TB) accounts for 1.4 million deaths annually and for one-fifth of adult deaths in poor/ low-income countries. Each patient with active pulmonary $\mathrm{TB}$, if left untreated, is estimated to infect 10-15 other individuals per year [2]. Thus, interrupting disease transmission is of major importance and requires early detection, in combination with adequate treatment.

\section{Host immune response in TB}

Depending on the immune response of the host, upon exposure to $M$. tuberculosis, individuals might eliminate the bacteria, progress to active TB or develop a persistent infection in the absence of clinical manifestation, referred to as latent TB. The initial response is due to the interaction of the unique lipids/ glycolipids/carbohydrates/peptidoglycans of the M. tuberculosis cell envelope, with cells of the innate immune system such as macrophages and dendritic cells [3]. The manner in which macrophages and dendritic cells activate or suppress distinct microbicidal mechanisms, the pattern of cytokine being produce and secreted, and how antigens interact with the major histocompatibility complex dictates the profile of the acquired immune response. The elicited acquired immune response mediated by T-cells plays a very important part in M. tuberculosis infection control [4]. However, the precise M. tuberculosis antigens and detailed profile of the host immune response necessary for effective acquired immunity to $M$. tuberculosis have yet to be determined. Most studies of the acquired immune response focus on the role played by antigenic proteins/peptides and very little address the mycobacterial antigens of a lipoglycan nature. However, lipoglycan antigens may have been undervalued and might in fact play a crucial part in the overall immune response to M. tuberculosis and as such be of extreme value for TB diagnosis, a subject discussed further ahead.

While the importance of T-cell immunity is long established, the role of humoral immunity has been considered controversial. There is however increasing recent evidence supporting a role for antibodies and B-cells in the establishment of an effective immune response against $M$. tuberculosis infection [5-7]. During active $\mathrm{TB}$, antibody responses are prominent [8, 9], and antibody levels to particular M. tuberculosis protein antigens may increase before symptoms of active TB [8]. Although people with active TB have been shown to produce antibodies with a low affinity to $M$. tuberculosis surface molecules and with a low ratio of IgG/IgM [10], there is evidence suggesting that specific antibodies might prevent M. tuberculosis dissemination. Antibodies in the mucosa may also potentially prevent infection via this route [11]. Elevated Ag85A-specific IgG titres have recently been identified as a correlate of a lower risk of TB disease in the MVA85A vaccine trial [12], indicating a possible role for antibodies in M. tuberculosis protective immunity. Here also, antibodies specific to mycobacterial glycolipids seem to play a relevant role [13-15] as discussed in detail later.

\section{The need for better TB diagnostic strategies and novel biomarkers}

Presently available markers/tests for TB diagnosis exhibit serious limitations, and none is a point-of-care (POC) diagnostic test. There is an intensive search for diagnostic biomarkers for TB [16-18], as well as predictive markers for progression from latent to active TB [19]. It is increasingly evident that latent TB should be viewed as part of a continuous spectrum, extending from sterilising immunity, to persistent nonprogressing infection and subclinical infection progressing to active disease [20,21]. Available tests are unable to distinguish those patients with subclinical progressing infection from those with nonprogressing latent infection [22-24]. Here we discuss the potential use of LAM in the diagnosis of active TB, in predicting the outcome following $M$. tuberculosis infection and in response to treatment.

Recently the World Health Organization (WHO) specified the target product profile (TPP) of the most urgently needed tests for TB [25]. With the objective of initiating treatment, the specificity should be $98 \%$ and for sensitivity the minimum requirements should be an overall sensitivity $\geqslant 65 \%$. Besides the need for a diagnostic test, WHO defined as one of the highest priorities a POC triage test, for systematic screening, able to distinguish individuals who need further confirmatory testing. The TPP for such a test was defined by WHO as overall specificity $>70 \%$ (optimally $>80 \%$ ) and sensitivity $>90 \%$ (optimally $>95 \%$ ). To achieve these goals the sensitivity of present tests should be increased, while retaining the high specificity. In addition, in line with the WHO goal of TB elimination by 2050, there is a great need for a test that identifies patients with latent $\mathrm{TB}$ who are on the rise of developing active disease.

Diagnostic laboratory methods for diagnosis of active TB are based on detection of evidence of M. tuberculosis by acid fast staining or direct culturing of bacilli in sputum specimens. Microscopy lacks sensitivity, while mycobacterial culturing, the ultimate diagnostic test for $\mathrm{TB}$, is a prolonged exercise that may require up to 6-8 weeks, or even more, to provide results. The GeneXpert MTB/RIF (Cepheid Inc. 
Sunnyvale, CA, USA), and more recently Xpert Ultra, a superior new generation version of GeneXpert, have become important additions to standard liquid culture.

Diagnosis of the latent forms of TB relies on the tuberculin skin test (TST) or the interferon- $\gamma$ release assays (IGRAs). The TST uses purified tuberculin derivative (PPD), which is nonspecific for M. tuberculosis. The interpretation of a TST needs to take into consideration false positive results in individuals exposed to ubiquitous non-TB mycobacteria and/or previously vaccinated with BCG. The commercial IGRAs (Quantiferon Gold-IT and more recently Quantiferon Plus (QIAGEN, Venlo, the Netherlands) as well as T-spot.TB (Oxford Immunotec, Marlborough, MA, USA) use M. tuberculosis-specific antigens (ESAT-6 and CFP-10) and include test tubes to control for anergy and background reactivity. However, neither the TST nor IGRAs can help identifying which of the patients with latent $\mathrm{TB}$ are on the verge of progression to active $\mathrm{TB}$, those in immunological control of M. tuberculosis nor the ones that exhibit just immunological memory to a previous $M$. tuberculosis infection. Thus, although these tests are useful in patient management they have no predictive value for progression to active TB [22-24, 26, 27].

Ideal diagnostic tests would distinguish between those with actual clinical TB; those with the potential reactivation/latent form; and those who have been cured. For the usage of the test in all different settings, less intrusive sources of potential biomarkers such as human urine, would be ideal.

An example of a new strategy that could be applied for both latent and active TB lies on the determination of particular circulating B lymphocytes in the peripheral blood [28]. It has been shown that B lymphocytes may discriminate individuals with latent and active $\mathrm{TB}$, namely the ratio of plasmablast to memory lymphocytes. Interestingly $\mathrm{Lu}$ et al. [29] reported recently that latent $\mathrm{TB}$ is associated with particular M. tuberculosis-specific antibody Fc functional profiles, and distinct antibody glycosylation patterns. Antibodies from patients with clinical evidence of TB when compared with those conforming to the definition of latent $\mathrm{TB}$, were found to drive phagolysosomal maturation, inflammasome activation, and macrophage killing of intracellular M. tuberculosis [29]. These results strongly suggest a role for Fc-mediated antibody effector functions for an effective immune response to M. tuberculosis [29].

Blood RNA signatures prospectively identified people at risk of developing active TB with a sensitivity of $53.7 \%$ (42.6-64.3) and a specificity of $82.8 \%$ (76.7-86.0) [30]. Interestingly, the same signature was later found in four different African countries [31]. This finding strongly validates the potential usefulness of the blood RNA signatures as an important tool to identify individuals at higher risk of progression to active disease.

A different strategy that holds promise is untargeted metabolomic approaches. However, the majority of metabolites in human body fluids that could accurately identify patients early in infection have lacked structural definition [32]; an intriguing exception is the identification of $N^{1}$-acetylisoputreanine an apparent novel polyamine metabolite in human urine that may have potential as a host-derived biomarker of M. tuberculosis infection [33].

In a proteomic approach to discover biomarkers for pulmonary TB in urine samples, 10 mycobacterial proteins were identified as unique biomarkers of the active from of TB whereas a different set of 6 proteins were characteristic of latent TB; partial identifications of these proteins were achieved, notably, isoforms of the PE-PGRS protein family were prominent in both the proteins from patients with active and latent TB [34].

\section{LAM as a biomarker for TB diagnosis}

LAM, a major lipoglycan (a multiglycosylated extension of the phosphatidylinositol mannoside (PIM) glycolipids) of the mycobacterial cell envelope has been shown to be present in the urine of M. tuberculosis-infected individuals.

LAM is a major constituent of the $M$. tuberculosis cell wall/envelope and represents up to $15 \%$ of the bacterial mass. It is composed of a mannan "core" (an extension of the PIM structure) which is decorated by a single branched arabinan chain. In addition, in the case of $M$. tuberculosis the majority of the terminal arabinose unity of the arabinan chain have attached to them short 2-4 mannose containing oligosaccharides called "mannose capping motifs"; this form of LAM is known as ManLAM [35-37] (figure 1). LAM exhibits important and distinct immunomodulatory properties [40, 41]. It is considered a virulence factor associated with the pathogenesis of $M$. tuberculosis infection. During mycobacterial infections, LAM is present in body fluids representing a potential biomarker to identify infected individuals. In addition, the immune response to LAM might also serve as a diagnostic tool. The usefulness of LAM as a diagnostic marker will be discussed in the following sections. 
FIGURE 1 Schematic rendition of the structure of Mycobacterium spp. LAM. Only the major common structural features are shown lsee various works for more structural detail [38, 39]). LAM: lipoarabinomannan.

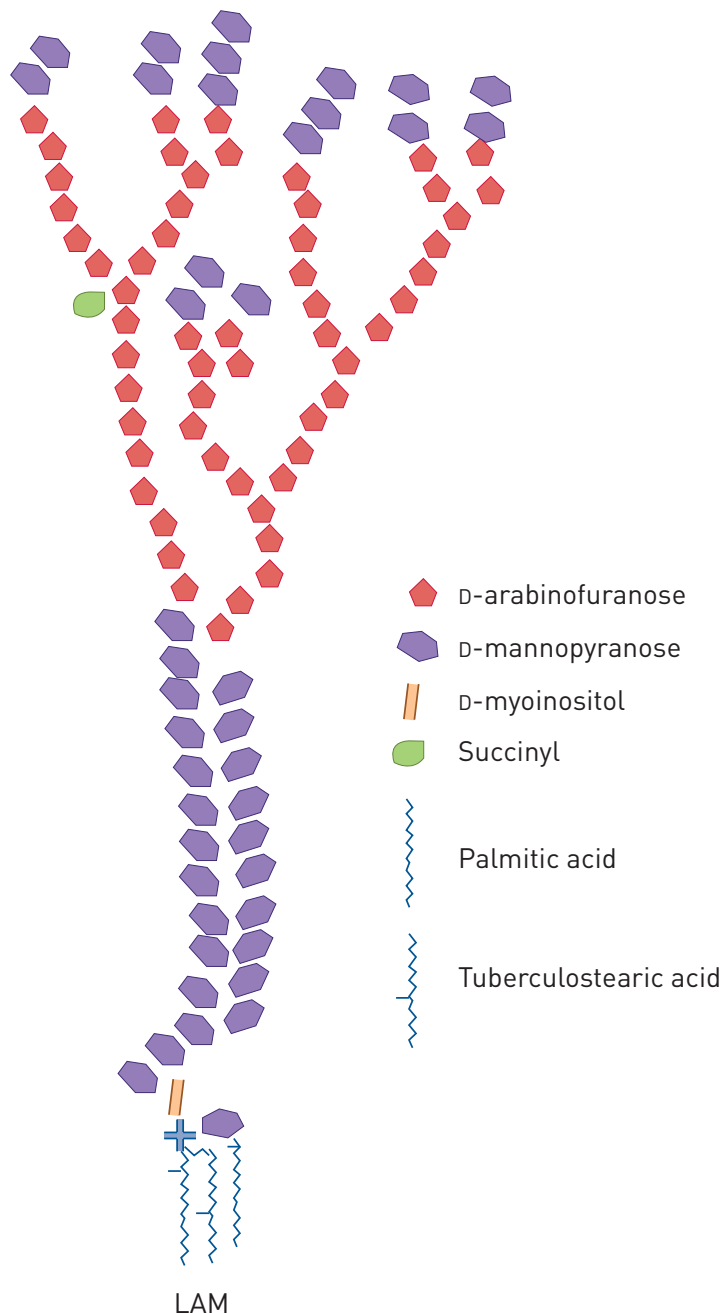

Detection of LAM in body fluids as a method for TB diagnosis

Tests that detect $M$. tuberculosis antigens in clinical specimens would potentially offer several advantages in terms of a specific biomarker of TB [42]. Tests for urinary LAM have generated great enthusiasm and numerous publications. However, several difficulties have been encountered over the last two decades and the consensus is that the present test(s) are not sensitive enough to diagnose latent TB nor active TB in HIV-negative patients [43-46]. However, recent results strongly suggest that this is a path that deserves further effort. A brief historical perspective will be presented followed by the results obtained with two commercially available tests and the efforts presently ongoing to render the use of LAM detection in urine, and potentially other body fluids, a useful diagnostic test for TB.

The first reports on the possible use of LAM in urine as a diagnostic test were published in 2001 by our team. We showed that LAM is present in urine from individuals with active TB [47-49] as well as mice injected with sonicated M. tuberculosis [47]. Although we clearly showed that LAM is present in urine of patients with active TB, the method used at that time required a laborious concentration step. The sensitivity achieved by us, as well as by several subsequent groups, did not reach the sensitivity required for a diagnostic test for TB.

Two tests were developed by companies and commercialised but obvious improvements are needed to render this approach of general use for the patients in need. A sandwich ELISA, employing polyclonal antibody preparations, was first marketed by Chemogen Inc. (South Portland, ME, USA) as the "Mtb ELISA" test and was sold as "Clearview TB ELISA" first by Inverness Medical Innovations and later by Alere Inc (Waltham, MA, USA). A set of clinical studies [50-55] using these commercial tests were performed, resulting in a pooled estimated sensitivity of $14 \%$ for patients with active TB and no HIV infection and $51 \%$ for patients co-infected with HIV (table 1) [56]. Subsequently a lateral flow test, the Determine TB-LAM Ag (Determine TB-LAM; Alere Inc.), using the same polyclonal antibody 


\begin{tabular}{lcc}
\hline \multicolumn{2}{l}{ TABLE 1 Performance of commercial urinary lipoarabinomannan (LAM) tests } & \\
& Sensitivity \% & Specificity \% \\
\hline $\begin{array}{l}\text { TB-LAM (Clearview) ELISA [56] } \\
\text { HIV-positive patients }\end{array}$ & $51(32-69)$ & $94(79-98)$ \\
HIV-negative patients & $14(7-24)$ & $97(90-99)$ \\
Determine (Alere) TB-LAM test [57] & $45(29-63)$ & $92(80-97)$ \\
HIV-positive patients & ND & ND \\
HIV-negative patients & & \\
\hline
\end{tabular}

preparations as in the Clearview ELISA, was produced as a POC test. Although easier to perform, the sensitivity of that test remained disappointingly low, as demonstrated in numerous clinical studies. In a systematic review [57] of 12 studies, 6 evaluated the Determine TB-LAM test to identify patients with active TB and 6 others evaluated the test for TB screening in HIV-positive adults. It was found that Determine TB-LAM has low sensitivity for both diagnosis and screening. The combination of Determine TB-LAM with sputum microscopy revealed an increased sensitivity for active TB when compared with either test alone but accompanied by a decreased specificity. Determine TB-LAM was shown to potentially improve the accuracy of active TB diagnosis only for seriously ill patients with HIV-co-infection and low $\mathrm{CD} 4^{+}$T-cell counts [57].

These tests were most sensitive for patients with profound immunosuppression revealing an inverse correlation between $\mathrm{CD}^{+}{ }^{+}$T-cell counts and urine LAM sensitivity [57-59] (figure 2). Pooled sensitivity of Determine TB-LAM was 56\% (41-70\%) and specificity $90 \%(81-95 \%)$ in participants with $\mathrm{CD}^{+}{ }^{+}$T-cell counts below or equal to 100 cells $\mu \mathrm{L}^{-1}$ [57] with a urinary LAM concentration in the range of ng per $\mathrm{mL}$ [60]. LAM positivity has been associated with poor prognosis [61] and with increased mortality [44, 62-65] and has been reported as an independent predictor of mortality [63]. In addition, the usage of bedside LAM testing as a guide for initiating anti-TB treatment in HIV-infected hospitalised inpatients has been associated with decreased mortality [66]. After a long evaluation process, WHO recommended in 2016 that the Determine TB-LAM assay might be utilised for TB diagnosis in a small subgroup of patients (i.e. in HIV-infected hospitalised adults with symptoms and signs of TB who either are seriously ill and/or have $\mathrm{CD} 4^{+} \mathrm{T}$-cell counts below or equal to 100 cells $\mu \mathrm{L}^{-1}$ ). Recently, in a randomised controlled trial of 2600 HIV-infected hospital inpatients with TB, the addition of urine LAM-based screening using the Alere TB-LAM test, a significant mortality reduction was observed in three subgroups of patients: those with low $\mathrm{CD} 4^{+} \mathrm{T}$-cell counts, severe anaemia or clinically suspected TB [67].

The high, and thus detectable, LAM concentration in urine of patients with advanced immunodeficiency has been postulated to depend on a high mycobacterial burden [68] and systemic dissemination of M. tuberculosis. It seems reasonable that patients with a more confined TB would excrete less LAM. Another interpretation could be that the renal tract is affected in advanced HIV infection with
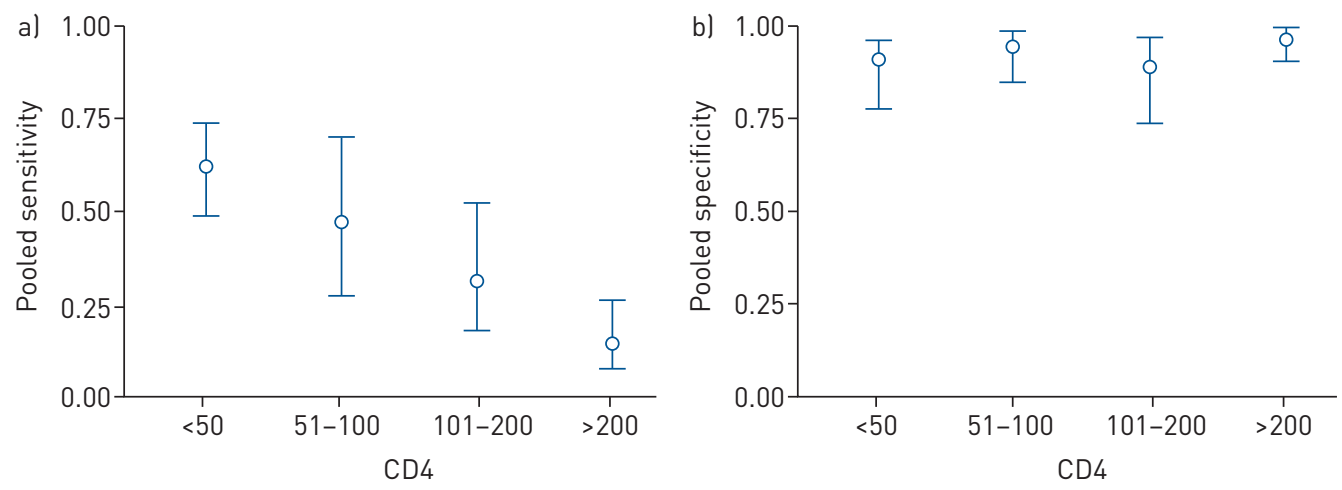

FIGURE 2 Correlation between $\mathrm{CD}^{+}$T-cell counts and lipoarabinomannan (LAM) detection in urine. Compilation of results from a meta-analysis of 12 different studies of tuberculosis (TB)-LAM for TB diagnosis and screening. Plots of a) sensitivity and b) specificity of TB-LAM stratified by CD4 ${ }^{+} \mathrm{T}$-cell counts. Circles represents the pooled estimates (median), with bars representing $95 \%$ credible intervals. Reproduced from [57] with permission from the publisher. 
accompanying mycobacteriuria [69-71]. More studies are required to reach consensus on this topic. The finding of LAM in urine prior to the initiation of antiretroviral therapy has also been reported to be associated with immune reconstitution inflammatory syndrome (IRIS) [60, 72], indicating that LAM detection in urine may predict the development of TB IRIS. The association of urinary LAM and IRIS supports the idea that high urinary LAM concentration reflects a disseminated disease with high bacterial load. Considering that LAM in urine might, at least in part, reflect the bacterial load in the body, measurement of LAM concentration has been suggested as a tool to monitor the effectiveness of anti-TB treatment (figure 3) [46, 64, 69].

Patients with latent or incipient TB who are on the verge of developing active TB, hypothetically carry bacteria that are metabolically active and dividing. Accordingly, one expects these individuals to excrete small amounts of LAM in urine, a hypothesis that needs to be tested with more clinical research. If confirmed, a more sensitive urinary LAM assay could possibly become a test to identify individuals at increased risk of progressing to active disease.

Detection of LAM in other body fluids has also been investigated. A few efforts have been made to detect LAM in sputum, without much success $[58,74]$, mainly depending on the viscosity and interfering matrix of the sputum. LAM was rarely detected in cerebrospinal fluids of patients with TB meningitis $[75,76]$ or in pleural effusions [77]. With respect to extrapulmonary $\mathrm{TB}$, tests for LAM in urine were rarely positive in one study of meningitis cases [78].

\section{Host immune responses to LAM as biomarkers for TB}

The source of LAM used in in vitro tests is of utmost importance for the correct dissection of the immune response to LAM. ManLAM derived from $M$. tuberculosis strain $\mathrm{H} 37 \mathrm{Rv}$ and clinical $M$. tuberculosis isolates elicit proinflammatory cytokine responses in human macrophages and dendritic cells $[3,79,80]$, while ManLAM from the laboratory strain M. tuberculosis Erdman induces no or just a weak response composed of pro- and anti-inflammatory cytokines [81]. Contamination with, for example, PIMs during the preparation of ManLAM may be the cause of some of the reports showing a weak proinflammatory response for particular ManLAM preparations [79]. Most studies of the adaptive immune response against $M$. tuberculosis have focused on the response to antigenic proteins/peptides and very little has been done on mycobacterial antigens of carbohydrate nature, in spite of the fact that mycobacterial glycolipids/lipoglycans are the dominant structural entities of the mycobacterial cell wall and have strong and distinct effects on the immune response. Only a few reports show the effect of LAM on the adaptive immune response. Liposomal delivery of LAM was shown to trigger $M$. tuberculosis-specific T-cell activation [82]; polycytotoxic T-cells from bronchiolar lavage were recently reported to be induced by mycobacterial glycolipids, in particular by LAM, which was associated with protection against M. tuberculosis infection [83].

Identifying specific markers or cytokine/chemokines of cell activation induced by LAM is an interesting venue to explore as an alternative to the present IGRA tests, in particular in the differentiation between

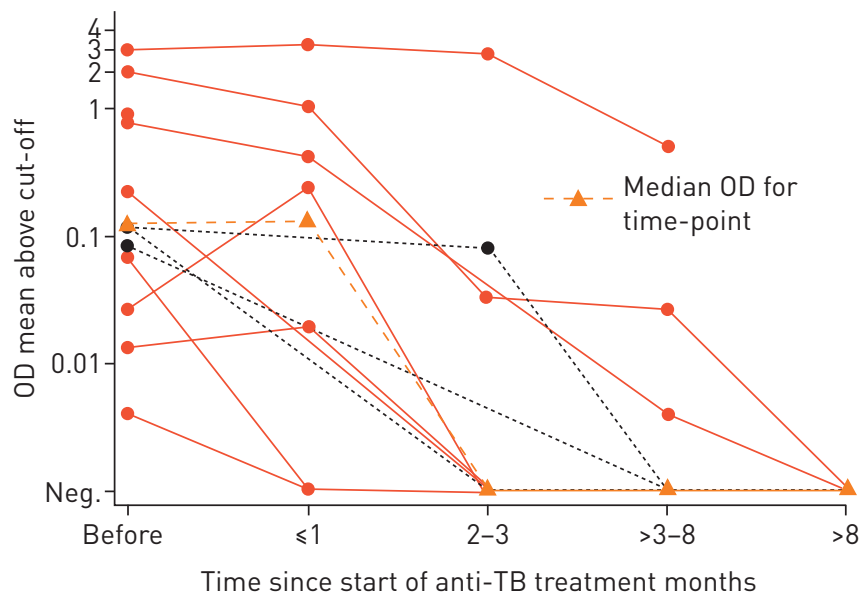

FIGURE 3 Signal intensity of Mycobacterium tuberculosis lipoarabinomannan in urine of children with tuberculosis (TB) in a high TB/HIV setting by ELISA at different time points after initiation of anti-TB treatment. Optical density (OD) results for HIV-positive participants are shown in red solid lines, results for HIV-negative participants as black dashed lines. The signal intensity became undetectable after 3 months of treatment in six participants. $y$-axis shows logarithmic scale for mean OD. Neg.: negative. Reproduced from [73]. 
different stages of latent TB as well as a complement for active TB diagnosis. Thus, there is an intense search for alternatives to ESAT-6 and CFP-10 as antigens used in the IGRA tests [84], as well as alternative to interferon (IFN) $-\gamma$ [85-87].

\section{Anti-LAM antibodies as markers of TB disease}

Anti-LAM antibodies are induced both during TB infection [88, 89] and after BCG vaccination [89-91]. These antibodies have been associated with $M$. tuberculosis opsonisation and restriction of intracellular growth $[90,91]$. Passive transfer of anti-LAM monoclonal antibodies has been shown to confer protection in mice $[13,14]$. Based on these findings a number of commercial tests based on antibody detection were developed [92], but none proved to be specific and sensitive enough. In a policy statement in 2011, WHO recommended against the usage of these tests while underscoring the relevance of continued investigation of diagnostic strategies based on antibody detection [93]. The majority of these commercial tests were directed at antibodies specific for protein antigens, except one, the Mycodot test (DynaGen Inc., Cambridge, MA, USA), that detects antibodies specific for LAM. Studies with the Mycodot test in Tanzania, Guinea Bissau and Japan revealed sensitivities and specificities from $16.0 \%$ to $67.5 \%$ and $84.0 \%$ to $97.5 \%$, respectively [94]. Using capsular arabinomannan, presumably devoid of the phosphatidyl inositol anchor and its constituent fatty acids, but otherwise structurally identical, anti-arabinomannan antibody titres were significantly higher for TB individuals that were HIV-negative than for the ones that were HIV co-infected in one study in Tanzania $(\mathrm{p}<0.01)$ [95]. In summary, the few studies performed using the Mycodot test indicate a high specificity (in contrast with serological tests for other, protein, antigens), but a low sensitivity. Although people with active TB have been shown to produce antibodies with a low affinity to the surface of live M. tuberculosis and with a low ratio of IgG/IgM [10], there is evidence suggesting that specific antibodies might prevent $M$. tuberculosis dissemination.

\section{Ongoing efforts to circumvent the technical limitations and render LAM detection in urine a useful test for TB patients}

Intensive efforts are presently being made by several research teams to develop a true POC test for TB diagnosis and patient management based on the simple detection and/or quantification of LAM in urine, with promising progress.

\section{Increasing the diagnostic sensitivity of urinary LAM tests}

Since the concentration of LAM in urine from HIV-negative adult TB patients is very low, in most cases, the analytical sensitivity of the method used is absolutely crucial. We, and others, have found that the true concentration of LAM in urine is in the range of $15 \mathrm{pg} \cdot \mathrm{mL}^{-1}$ to several hundred $\mathrm{ng} \cdot \mathrm{mL}^{-1}[60,96-98]$. Based on our findings that urine concentration renders LAM detectable for most "ordinary" TB patients [47], we developed a simple concentration method. This was based on very efficient monoclonal anti-LAM antibodies in combination with a concentration step using gold coated nanoparticles [97]. With this method, we reached a sensitivity of $82 \%$ in HIV-negative patients with active TB. However, some of the components of the test were not stable enough, resulting in a shelf life of the test of about 3 months. Second, as shown next, when the analytical sensitivity increased, the "matrix" effect of the urine became more prominent (i.e. the LAM signal was masked apparently by as yet unidentified components in the urine).

Other approaches to increase the sensitivity and/or the simplicity of the test have been made [99], namely: 1) immunoassays exploring single-molecule fluorescence-linked immunosorbent assay [100]; 2) waveguide-based optical biosensors [101]; or 3) electrochemical detection on a disposable chip [102]. Efforts to engineer new anti-LAM antibodies with higher affinity have for instance resulted in recombinant antibodies [103]. None of these approaches have so far reached sufficient sensitivity for clinical use. More recently a method applying a copper complex dye within a hydrogel nanocage that captures LAM was developed, and a sensitivity of $96 \%$ and specificity of $81 \%$ for diagnosis of pulmonary TB disease in HIV-negative adults was reported [104].

\section{Matrix effect and how to overcome it}

In experimental studies we found that urine from TB patients and healthy individuals inhibit LAM detection at varying degrees, when purified LAM was added to different urine samples. Data shown in figure 4, using a set of 15 urine samples, clearly shows that the matrix effect varies between urine samples, LAM in the spiked urine being detectable for some individuals (see for example samples 1, 2, 9, 100 and 11 in figure 4) while for others it is undetectable (see for example samples 3 and 4 in figure 4). The inhibitory effect ("matrix" effect) varied not only between individuals, but also between samples taken at various time points from the same individual (data not shown).

The matrix effect is extremely important for the measurement of urine analytes. The composition of human urine is highly variable [105] with large variations in concentration of individual components [106, 107], 


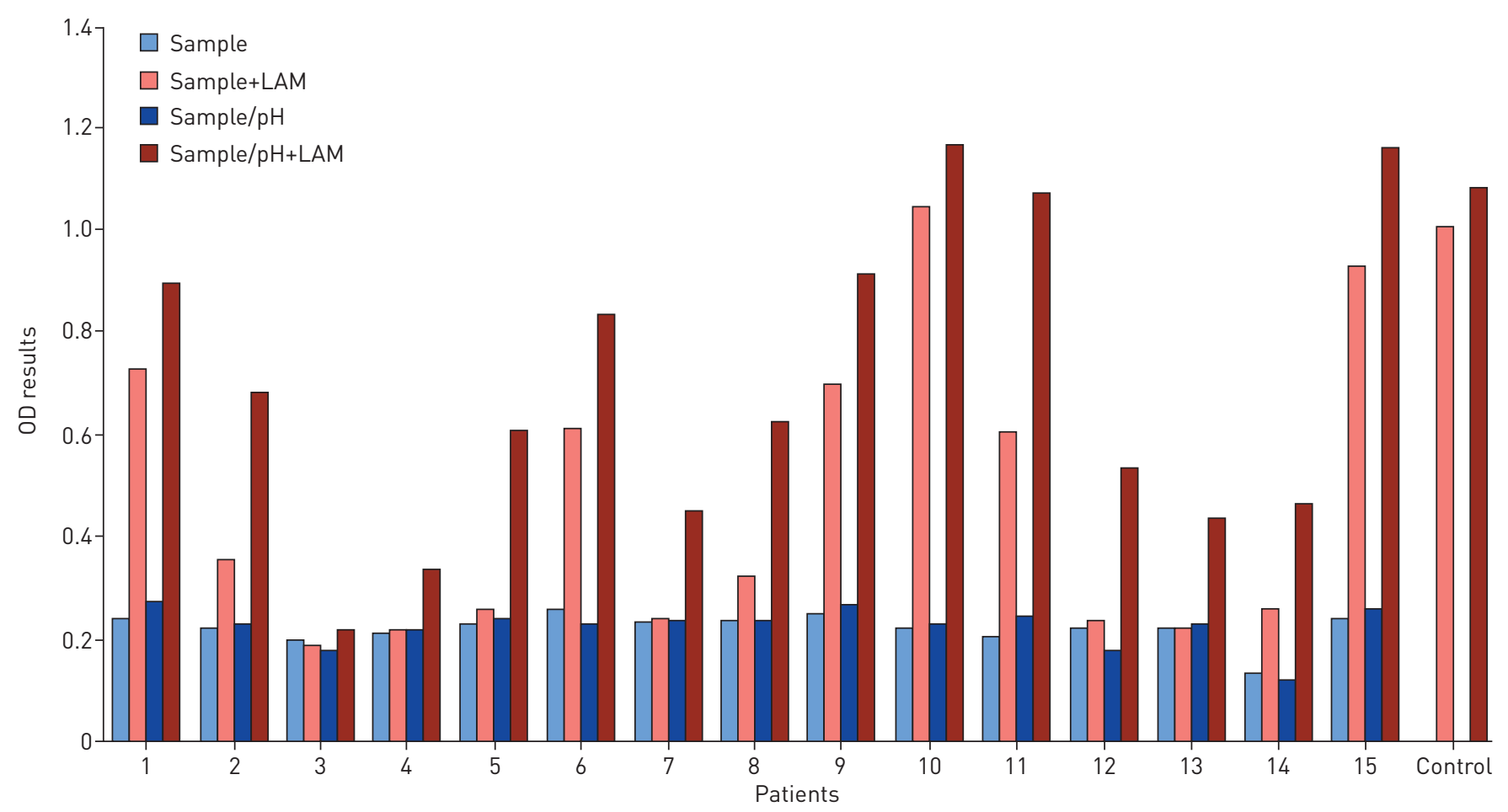

FIGURE 4 Lipoarabinomannan (LAM) signal by ELISA in urine samples from children with suspected tuberculosis. Samples were collected at Machava General Hospital in Maputo, Mozambique, and stored at $-80^{\circ} \mathrm{C}$. The samples were transported frozen to the laboratory at Karolinska Institutet, Sweden. After thawing each urine was divided into four samples: 1) nonmanipulated; 2) spiked with $250 \mathrm{pg} \cdot \mathrm{mL}^{-1} \mathrm{LAM}$; 3) pH adjusted with $40 \mathrm{mM}$ Tris final concentration; 4) pH adjusted and spiked with LAM. LAM signal was measured by ELISA with absorbance at $450 \mathrm{~nm}$ following the protocol described previously [97]. PBS spiked with $250 \mathrm{pg} \cdot \mathrm{mL}^{-1}$ LAM was used as a control. LAM was prepared in house as described previously [79].

containing both organic and inorganic constituents, such as carbohydrates, amino acids, peptides, lipids and end products of metabolism [108]. These organic entities of the urine matrix, as well as $\mathrm{pH}$ and electrolytes, vary depending on normal physiological variations, health conditions, as well as differences in food consumption and medication intake. In immunoassays the matrix components may mask and/or change the conformation of either antibodies or LAM epitopes in the assay. This masking effect may vary depending on the antibody and/or of LAM epitopes detected by distinct antibodies as well as the type of assay used [109]. Reducing the inhibitory effects of patient urines obviously would increase the diagnostic sensitivity and accuracy of a LAM-based urine test.

The matrix effect on the LAM signal is evident also in serum [110]. The matrix effect in the serum has been considered to depend on the faculty of ManLAM to form complexes with antibodies [111] and other proteins. Using spiked samples, SAKAMURI et al. [112] demonstrated that LAM forms complexes with high-density lipoprotein present in the serum. In the case of serum various methods of pre-treatment have been tried to overcome the matrix effect using different pre-treatment schemes designed to disrupt complexing with proteins and other components in serum and shown to improve detection $[110,113]$. Perchloric acid-based pre-treatment was shown to significantly improve the ability to detect LAM [110].

The importance of $\mathrm{pH}$

Low $\mathrm{pH}$ most often occurs in malnutrition [114] such as wasting TB disease and might reduce the ability to detect LAM. We found that adjusting the $\mathrm{pH}$ to a neutral level had some positive effect on the LAM signal. Data depicted in figure 4 reveal that adjusting the $\mathrm{pH}$ tends to improve LAM detection and that in some cases is sufficient to render LAM detectable (see for example patients 5, 7, 8 and 12 in figure 4), However, the differences in urinary $\mathrm{pH}$ do not exclusively explain the large variations in LAM recovery between different urine samples.

\section{Effect of urine concentration on LAM detection}

We, and others, have postulated that the more the urine was concentrated the higher would be the LAM signal. Indeed, in a recent study, GiNA et al. [115] showed that collection of early morning urine (that is more concentrated) improves the urinary lateral flow LAM assay sensitivity in hospitalised patients with 
FIGURE 5 Effect of storage of urine at $4^{\circ} \mathrm{C}$ on lipoarabinomannan (LAM) signal detected by ELISA. Urine was spiked with various concentrations of LAM and stored at $4^{\circ} \mathrm{C}$. LAM signal was measured by ELISA with absorbance at $450 \mathrm{~nm}$ following the protocol described previously [97].

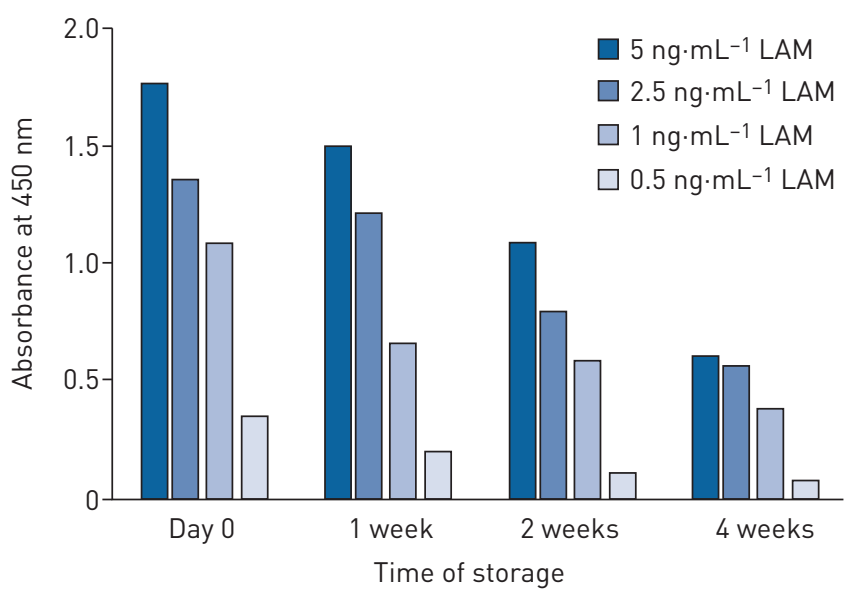

TB and HIV-coinfection. However, we found that the higher the concentration of urinary creatinine, which is a marker of urine concentration, the lower the signal (data not shown), indicating that more concentrated urine contains more inhibitory factors. So, it seems that in patients with TB/HIV, co-infection and presumably higher concentrations of LAM in urine, the matrix does not mask the LAM signal while in HIV-negative patients (presumably with low LAM concentration) the matrix effect is enforced by concentration. We thus tested the hypothesis that diluting urine would reduce the matrix effect on the LAM signal, a standard method for avoiding the matrix effect in biological assays [107]. Indeed, we found that the LAM signal is increased in diluted urine spiked with LAM (data not shown). However, dilution of samples with very low concentration of excreted LAM is ineffective since LAM is diluted to levels below detection limits.

\section{Effect of sample transportation and storage}

Since transportation and storage of urine samples is a key issue in clinical studies where testing is centralised, we investigated the impact of various storage temperatures on the LAM signal in spiked urine samples. We, and others [101], found that when the samples are stored at room temperature the signal decreases rapidly, in a matter of hours. Even storage at $4^{\circ} \mathrm{C}$ causes a significant decrease in the LAM detection as shown in figure 5 for periods as short as 1,2 and 4 weeks. These findings are in agreement with a study showing that after storage of urine for $2 \mathrm{~h}$ at room temperature LAM becomes undetectable using an immunoblot method [101]. We, and others [116], also found that freeze-thawing extensively reduces LAM detection.

Actually, the fate of carbohydrate containing molecules may also be affected by chemical reactions in the urine. Thus, the formation of glucose ureide by the interaction of glucose with urea results in ammonia, an event which may change the $\mathrm{pH}$ of urine. Urea in urine can, depending on the time of exposure, temperature and $\mathrm{pH}$, disintegrate into isocyanates that may interact with amines on the antibodies, thus potentially interfering with the binding of the antibodies with carbohydrates like LAM.

\section{TABLE 2 Lipoarabinomannan as a potential biomarker for tuberculosis diagnosis}

\begin{tabular}{|c|c|c|}
\hline Type of test & Present status & To be explored \\
\hline Urine antigen detection & $\begin{array}{l}\text { Low sensitivity, high specificity } \\
\text { Useful in subgroup of HIV-positive }\end{array}$ & $\begin{array}{l}\text { Solve matrix problems } \\
\text { Increase sensitivity } \\
\text { Generate a true POC test }\end{array}$ \\
\hline Serum antigen detection & $\begin{array}{l}\text { Matrix problems } \\
\text { Immune complexes }\end{array}$ & $\begin{array}{l}\text { Solve matrix and immune complex } \\
\text { problems }\end{array}$ \\
\hline Cellular immune response & $\begin{array}{c}\text { Considered a potential alternative to } \\
\text { present IGRA }\end{array}$ & $\begin{array}{c}\text { Marker of protection (polycytotoxic } \\
\text { T-cells) }\end{array}$ \\
\hline Humoral immune response & Low sensitivity, high specificity & $\begin{array}{c}\text { Usefulness for specific subgroups } \\
\text { Marker of protection }\end{array}$ \\
\hline
\end{tabular}




\section{Concluding remarks}

There are several interesting venues for the use of LAM as a biomarker in the diagnosis of TB (table 2), in particular the use of a urinary LAM-based POC test. To achieve this goal, a simple concentration step is needed in combination with a method to circumvent the matrix effect of the urine. A method for detection, or even quantification, of LAM in serum should be further explored.

If increased sensitivity of an antigen test (urine or serum) for LAM is achieved, LAM should be explored as a predictive biomarker of the outcomes following $M$. tuberculosis infection, as well as a marker to evaluate the efficacy of anti-TB therapies.

Markers for host response to LAM (cellular and humoral) should be explored for a possible prognostic test to identify those at highest risk of progressing to active TB.

Conflict of interest: M. Correia-Neves has nothing to disclose. G. Fröberg has nothing to disclose. L. Korshun has nothing to disclose. S. Viegas has nothing to disclose. P. Vaz reports receiving grants from the Ariel Foundation during the conduct of the study. N. Ramanlal reports receiving grants from the Ariel Foundation during the conduct of the study. J. Bruchfeld has nothing to disclose. B. Hamasur has nothing to disclose. P. Brennan has nothing to disclose. G. Källenius reports receiving grants from the Optimus Foundation during the conduct of the study and owns shares in TBDiadirect $\mathrm{AB}$

\section{References}

1 Houben RM, Dodd PJ. The global burden of latent tuberculosis infection: a re-estimation using mathematical modelling. PLoS Med 2016; 13: e1002152.

2 WHO. Global Tuberculosis Report. 2016.

3 Kallenius G, Correia-Neves M, Buteme H, et al. Lipoarabinomannan, and its related glycolipids, induce divergent and opposing immune responses to Mycobacterium tuberculosis depending on structural diversity and experimental variations. Tuberculosis (Edinb) 2016; 96: 120-130.

4 Cooper AM. Cell-mediated immune responses in tuberculosis. Annu Rev Immunol 2009; 27: 393-422.

5 Achkar JM, Chan J, Casadevall A. B cells and antibodies in the defense against Mycobacterium tuberculosis infection. Immunol Rev 2015; 264: 167-181.

6 Kozakiewicz L, Phuah J, Flynn J, et al. The role of B cells and humoral immunity in Mycobacterium tuberculosis infection. Adv Exp Med Biol 2013; 783: 225-250.

7 Jacobs AJ, Mongkolsapaya J, Screaton GR, et al. Antibodies and tuberculosis. Tuberculosis (Edinb) 2016; 101: $102-113$.

8 Gennaro ML, Affouf M, Kanaujia GV, et al. Antibody markers of incident tuberculosis among HIV-infected adults in the USA: a historical prospective study. Int J Tuberc Lung Dis 2007; 11: 624-631.

9 Laal S, Samanich KM, Sonnenberg MG, et al. Human humoral responses to antigens of Mycobacterium tuberculosis: immunodominance of high-molecular-mass antigens. Clin Diagn Lab Immunol 1997; 4: 49-56.

10 Perley CC, Frahm M, Click EM, et al. The human antibody response to the surface of Mycobacterium tuberculosis. PLoS One 2014; 9: e98938.

11 Alvarez N, Otero O, Camacho F, et al. Passive administration of purified secretory IgA from human colostrum induces protection against Mycobacterium tuberculosis in a murine model of progressive pulmonary infection. BMC Immunol 2013; 14: Suppl. 1, S3.

12 Fletcher HA, Snowden MA, Landry B, et al. T-cell activation is an immune correlate of risk in BCG vaccinated infants. Nat Commun 2016; 7: 11290.

13 Hamasur B, Haile M, Pawlowski A, et al. A mycobacterial lipoarabinomannan specific monoclonal antibody and its $\mathrm{F}\left(\mathrm{ab}^{\prime}\right)$ fragment prolong survival of mice infected with Mycobacterium tuberculosis. Clin Exp Immunol 2004; 138: 30-38.

14 Teitelbaum R, Glatman-Freedman A, Chen B, et al. A mAb recognizing a surface antigen of Mycobacterium tuberculosis enhances host survival. Proc Natl Acad Sci USA 1998; 95: 15688-15693.

15 Cardona PJ, Julian E, Valles X, et al. Production of antibodies against glycolipids from the Mycobacterium tuberculosis cell wall in aerosol murine models of tuberculosis. Scand J Immunol 2002; 55: 639-645.

16 Weiner J, Kaufmann SH. High-throughput and computational approaches for diagnostic and prognostic host tuberculosis biomarkers. Int J Infect Dis 2017; 56: 258-262.

17 Goletti D, Petruccioli E, Joosten SA, et al. Tuberculosis biomarkers: from diagnosis to protection. Infect Dis Rep 2016; 8: 6568 .

18 Wallis RS, Doherty TM, Onyebujoh P, et al. Biomarkers for tuberculosis disease activity, cure, and relapse. Lancet Infect Dis 2009; 9: 162-172.

19 Petruccioli E, Scriba TJ, Petrone L, et al. Correlates of tuberculosis risk: predictive biomarkers for progression to active tuberculosis. Eur Respir J 2016; 48: 1751-1763.

20 Barry CE III, Boshoff HI, Dartois V, et al. The spectrum of latent tuberculosis: rethinking the biology and intervention strategies. Nat Rev Microbiol 2009; 7: 845-855.

21 Salgame P, Geadas C, Collins L, et al. Latent tuberculosis infection: revisiting and revising concepts. Tuberculosis (Edinb) 2015; 95: 373-384.

22 Pai M, Denkinger CM, Kik SV, et al. Gamma interferon release assays for detection of Mycobacterium tuberculosis infection. Clin Microbiol Rev 2014; 27: 3-20.

23 Auguste P, Tsertsvadze A, Pink J, et al. Comparing interferon-gamma release assays with tuberculin skin test for identifying latent tuberculosis infection that progresses to active tuberculosis: systematic review and meta-analysis. BMC Infect Dis 2017; 17: 200.

24 Nemes E, Rozot V, Geldenhuys H, et al. Optimization and interpretation of serial QuantiFERON testing to measure acquisition of Mycobacterium tuberculosis infection. Am J Respir Crit Care Med 2017; 196: 638-648. 
56 Minion J, Leung E, Talbot E, et al. Diagnosing tuberculosis with urine lipoarabinomannan: systematic review and meta-analysis. Eur Respir J 2011; 38: 1398-1405

57 Shah M, Hanrahan C, Wang ZY, et al. Lateral flow urine lipoarabinomannan assay for detecting active tuberculosis in HIV-positive adults. Cochrane Database Syst Rev 2016; 5: CD011420.

58 Dheda K, Davids V, Lenders L, et al. Clinical utility of a commercial LAM-ELISA assay for TB diagnosis in HIV-infected patients using urine and sputum samples. PLoS One 2010; 5: e9848.

59 Swaminathan S, Rekha VV. Antigen detection as a point-of-care test for TB: the case of lipoarabinomannan Future Microbiol 2012; 7: 559-564.

WHO. High-priority target product profiles for new tuberculosis diagnostics. Report of a consensus meeting. 2014. , Hawkridge $T$, Verver S, et al. The tuberculin skin test versus QuantiFERON TB Gold(R) in of QuantiFERON conversions. Am J Respir Crit Care Med 2017; 196: 546-547.

Sebina I, Biraro IA, Dockrell HM, et al. Circulating B-lymphocytes as potential biomarkers of tuberculosis

(

tuberculosis. Am J Respir Crit Care Med 2018; 197: 1198-1208. treatment. BMC Infect Dis 2014; 14: 53 .

Fitzgerald BL, Mahapatra S, Farmer DK, et al. Elucidating the structure of N1-acetylisoputreanine: a nove Eur Respir J 2014; 43: 1719-1729. lipoarabinomannan of Mycobacterium tuberculosis. I Biol Chem 1991; 266: 9652-9660. and lipomannan of Mycobacterium tuberculosis. I Biol Chem 1990; 265: 9272-9279. Mycobacterium avium. I Biol Chem 1988; 263: 4092-4097.

Kaur D, Angala SK, Wu SW, et al. A single arabinan chain is attached to the phosphatidylinositol mannosyl core of the major immunomoc wall core. I Biol Chem 2016; 291: 18867-18879. antigens from the leprosy and tubercle bacilli. I Biol Chem 1986; 261: 12345-12351.

to modulation of the immune response. Mol Microbiol 2004; 53: 391-403. the diagnosis of tuberculosis. Clin Vaccine Immunol 2011; 18: 1616-1627.

Sahle SN, Asress DT, Tullu KD, et al. Performance of point-of-care urine test in diagnosing tuberculosis suspects with and with

(LAM) in diagnosing tuberculosis and predicting mortality with and without HIV: prospective TB cohort from the (B) in children: a prospective study. Lancet Glob Health 2014; 2: e278-e284.

Tanzania. Eur Respir J 2015; 46: 761-770. lipoarabi B, Bruchfeld J, Haile M, et al. tuberculosis centre. Scand I Infect Dis 2001; 33: 279-284. lipoarabinomannan in Ethiopian tuberculosis patients. Scand I Infect Dis 2002; 34: 167-171. 99: 893-900.

detection in African tuberculosis suspects and patients. Int J Tuberc Lung Dis 2009; 13: 1253-1259. tuberculosis. BMC Infect Dis 2009; 9: 141 . hospitalized patients in a High HIV prevalence setting. I Acquir Immune Defic Syndr 2009; 52: 145-151. ( immunosorbent assay for screening ambulatory HIV-infected persons for tuberculosis. J Acquir Immune Defic 
Conesa-Botella A, Loembe MM, Manabe YC, et al. Urinary lipoarabinomannan as predictor for the tuberculosis immune reconstitution inflammatory syndrome. J Acquir Immune Defic Syndr 2011; 58: 463-468.

Lawn SD, Kerkhoff AD, Burton R, et al. Diagnostic accuracy, incremental yield and prognostic value of Determine TB-LAM for routine diagnostic testing for tuberculosis in HIV-infected patients requiring acute hospital admission in South Africa: a prospective cohort. BMC Med 2017; 15: 67.

Balcha TT, Winqvist N, Sturegard E, et al. Detection of lipoarabinomannan in urine for identification of active tuberculosis among HIV-positive adults in Ethiopian health centres. Trop Med Int Health 2014; 19: 734-742.

Gupta-Wright A, Peters JA, Flach C, et al. Detection of lipoarabinomannan (LAM) in urine is an independent predictor of mortality risk in patients receiving treatment for HIV-associated tuberculosis in sub-Saharan Africa: a systematic review and meta-analysis. BMC Med 2016; 14: 53.

Drain PK, Gounder L, Grobler A, et al. Urine lipoarabinomannan to monitor anti-tuberculosis therapy response and predict mortality in an HIV-endemic region: a prospective cohort study. BMJ Open 2015; 5: e006833.

Manabe YC, Nonyane BA, Nakiyingi L, et al. Point-of-care lateral flow assays for tuberculosis and cryptococcal antigenuria predict death in HIV infected adults in Uganda. PLoS One 2014; 9: e101459.

Peter JG, Zijenah LS, Chanda D, et al. Effect on mortality of point-of-care, urine-based lipoarabinomannan testing to guide tuberculosis treatment initiation in HIV-positive hospital inpatients: a pragmatic, parallel-group, multicountry, open-label, randomised controlled trial. Lancet 2016; 387: 1187-1197.

Gupta-Wright A, Corbett EL, van Oosterhout JJ, et al. Rapid urine-based screening for tuberculosis in HIV-positive patients admitted to hospital in Africa (STAMP): a pragmatic, multicentre, parallel-group, double-blind, randomised controlled trial. Lancet 2018; 392: 292-301.

Nakiyingi L, Ssengooba W, Nakanjako D, et al. Predictors and outcomes of mycobacteremia among HIV-infected smear- negative presumptive tuberculosis patients in Uganda. BMC Infect Dis 2015; 15: 62.

Wood R, Racow K, Bekker LG, et al. Lipoarabinomannan in urine during tuberculosis treatment: association with host and pathogen factors and mycobacteriuria. BMC Infect Dis 2012; 12: 47.

Cox JA, Lukande RL, Kalungi S, et al. Is urinary lipoarabinomannan the result of renal tuberculosis? Assessment of the renal histology in an autopsy cohort of Ugandan HIV-Infected adults. PLoS One 2015; 10: e0123323.

Lawn SD, Gupta-Wright A. Detection of lipoarabinomannan (LAM) in urine is indicative of disseminated TB with renal involvement in patients living with HIV and advanced immunodeficiency: evidence and implications. Trans R Soc Trop Med Hyg 2016; 110: 180-185.

Lawn SD, Edwards DJ, Kranzer K, et al. Urine lipoarabinomannan assay for tuberculosis screening before antiretroviral therapy diagnostic yield and association with immune reconstitution disease. AIDS 2009; 23: $1875-1880$.

Kroidl I, Clowes P, Reither K, et al. Performance of urine lipoarabinomannan assays for paediatric tuberculosis in Tanzania. Eur Respir J 2015; 46: 761-770.

Peter JG, Cashmore TJ, Meldau R, et al. Diagnostic accuracy of induced sputum LAM ELISA for tuberculosis diagnosis in sputum-scarce patients. Int J Tuberc Lung Dis 2012; 16: 1108-1112.

Patel VB, Bhigjee AI, Paruk HF, et al. Utility of a novel lipoarabinomannan assay for the diagnosis of tuberculous meningitis in a resource-poor high-HIV prevalence setting. Cerebrospinal Fluid Res 2009; 6: 13.

Bahr NC, Tugume L, Boulware DR. A word of caution in considering the use of the lipoarabinomannan lateral flow assay on cerebrospinal fluid for detection of tuberculous meningitis. J Clin Microbiol 2016; 54: 241-242.

Dheda K, Van-Zyl Smit RN, Sechi LA, et al. Clinical diagnostic utility of IP-10 and LAM antigen levels for the diagnosis of tuberculous pleural effusions in a high burden setting. PLoS One 2009; 4: e4689.

Blok N, Visser DH, Solomons R, et al. Lipoarabinomannan enzyme-linked immunosorbent assay for early diagnosis of childhood tuberculous meningitis. Int J Tuberc Lung Dis 2014; 18: 205-210.

Mazurek J, Ignatowicz L, Kallenius G, et al. Divergent effects of mycobacterial cell wall glycolipids on maturation and function of human monocyte-derived dendritic cells. PLoS One 2012; 7: e42515.

Yonekawa A, Saijo S, Hoshino Y, et al. Dectin-2 is a direct receptor for mannose-capped lipoarabinomannan of mycobacteria. Immunity 2014; 41: 402-413.

Bradbury MG, Moreno C. Effect of lipoarabinomannan and mycobacteria on tumour necrosis factor production by different populations of murine macrophages. Clin Exp Immunol 1993; 94: 57-63.

Kallert S, Zenk SF, Walther P, et al. Liposomal delivery of lipoarabinomannan triggers Mycobacterium tuberculosis specific T-cells. Tuberculosis (Edinb) 2015; 95: 452-462.

Busch M, Herzmann C, Kallert S, et al. Lipoarabinomannan-responsive polycytotoxic t cells are associated with protection in human tuberculosis. Am J Respir Crit Care Med 2016; 194: 345-355.

Chegou NN, Black GF, Kidd M, et al. Host markers in QuantiFERON supernatants differentiate active TB from latent TB infection: preliminary report. BMC Pulm Med 2009; 9: 21.

Frahm M, Goswami ND, Owzar K, et al. Discriminating between latent and active tuberculosis with multiple biomarker responses. Tuberculosis (Edinb) 2011; 91: 250-256.

Jeong YH, Hur YG, Lee $\mathrm{H}$, et al. Discrimination between active and latent tuberculosis based on ratio of antigen-specific to mitogen-induced IP-10 production. J Clin Microbiol 2015; 53: 504-510.

Nonghanphithak D, Reechaipichitkul W, Namwat W, et al. Chemokines additional to IFN-gamma can be used to differentiate among Mycobacterium tuberculosis infection possibilities and provide evidence of an early clearance phenotype. Tuberculosis (Edinb) 2017; 105: 28-34.

Tessema TA, Bjune G, Hamasur B, et al. Circulating antibodies to lipoarabinomannan in relation to sputum microscopy, clinical features and urinary anti-lipoarabinomannan detection in pulmonary tuberculosis. Scand J Infect Dis 2002; 34: 97-103.

Brown RM, Cruz O, Brennan M, et al. Lipoarabinomannan-reactive human secretory immunoglobulin A responses induced by mucosal bacille Calmette-Guerin vaccination. J Infect Dis 2003; 187: 513-517.

de Valliere S, Abate G, Blazevic A, et al. Enhancement of innate and cell-mediated immunity by antimycobacterial antibodies. Infect Immun 2005; 73: 6711-6720.

Chen T, Blanc C, Eder AZ, et al. Association of human antibodies to arabinomannan with enhanced mycobacterial opsonophagocytosis and intracellular growth reduction. J Infect Dis 2016; 214: 300-310. 
Steingart KR, Flores LL, Dendukuri N, et al. Commercial serological tests for the diagnosis of active pulmonary and extrapulmonary tuberculosis: an updated systematic review and meta-analysis. PLoS Med 2011; 8: e1001062. WHO. Commercial sero-diagnostic tests for diagnosis of tuberculosis: policy statement. 2011.

Somi GR, O’Brien RJ, Mfinanga GS, et al. Evaluation of the MycoDot test in patients with suspected tuberculosis in a field setting in Tanzania. Int J Tuberc Lung Dis 1999; 3: 231-238.

Yu X, Prados-Rosales R, Jenny-Avital ER, et al. Comparative evaluation of profiles of antibodies to mycobacterial capsular polysaccharides in tuberculosis patients and controls stratified by HIV status. Clin Vaccine Immunol 2012; 19: 198-208.

Savolainen L, Kantele A, Sandboge B, et al. Modification of Clearview tuberculosis (TB) enzyme-linked immunosorbent assay for TB patients not infected with HIV. Clin Vaccine Immunol 2013; 20: 1479-1482.

, van Helden P, et al. A sensitive urinary lipoarabinomannan test for tuberculosis. PLoS One 2015; 10: e0123457.

De P, Amin AG, Valli E, et al. Estimation of D-arabinose by gas chromatography/mass spectrometry as surrogate for mycobacterial lipoarabinomannan in human urine. PLoS One 2015; 10: e0144088.

Sarkar S, Tang XL, Das D, et al. A bispecific antibody based assay shows potential for detecting tuberculosis in resource constrained laboratory settings. PLoS One 2012; 7: e32340.

Schmidt R, Jacak J, Schirwitz C, et al. Single-molecule detection on a protein-array assay platform for the exposure of a tuberculosis antigen. J Proteome Res 2011; 10: 1316-1322. sandwich immunoassay format using a waveguide-based optical biosensor. Tuberculosis (Edinb) 2012; 92: 407-416.

Wang L, Leng C, Tang S, et al. Enzyme-free signal amplification for electrochemical detection of Mycobacterium lipoarabinomannan antibody on a disposable chip. Biosens Bioelectron 2012; 38: 421-424.

M. tuberculosis. Sci Rep 2015; 5: 10281.

Paris L, Magni R, Zaidi F, et al. Urine lipoarabinomannan glycan in HIV-negative patients with pulmonary tuberculosis correlates with disease severity. Sci Transl Med 2017; 9: eaal2807. 404: 140-143.

Simerville JA, Maxted WC, Pahira JJ. Urinalysis: a comprehensive review. Am Fam Physician 2005; 71: 1153-1162.

Taylor TP, Janech MG, Slate EH, et al. Overcoming the effects of matrix interference in the measurement of urine protein analytes. Biomark Insights 2012; 7: 1-8.

Bouatra S, Aziat F, Mandal R, et al. The human urine metabolome. PLoS One 2013; 8: e73076.

Boscato LM, Stuart MC. Heterophilic antibodies: a problem for all immunoassays. Clin Chem 1988; 34: 27-33.

Laurentius LB, Crawford AC, Mulvihill TS, et al. Importance of specimen pretreatment for the low-level detection of mycobacterial lipoarabinomannan in human serum. Analyst 2016; 142: 177-185.

Bhattacharya A, Ranadive SN, Kale M, et al. Antibody-based enzyme-linked immunosorbent assay for determination of immune complexes in clinical tuberculosis. Am Rev Respir Dis 1986; 134: 205-209.

Sakamuri RM, Price DN, Lee M, et al. Association of lipoarabinomannan with high density lipoprotein in blood: implications for diagnostics. Tuberculosis (Edinb) 2013; 93: 301-307.

Crawford AC, Laurentius LB, Mulvihill TS, et al. Detection of the tuberculosis antigenic marker mannose-capped lipoarabinomannan in pretreated serum by surface-enhanced Raman scattering. Analyst 2016; 142: $186-196$. 325-331

Gina P, Randall PJ, Muchinga TE, et al. Early morning urine collection to improve urinary lateral flow LAM assay sensitivity in hospitalised patients with HIV-TB co-infection. BMC Infect Dis 2017; 17: 339. tuberculosis: recommendations on the design and reporting of clinical studies. BMC Infect Dis 2013; 13 : 407. 